\title{
Mixed Convection in a Vertical Porous Channel
}

\author{
J. C. Umavathi · J. P. Kumar · A. J. Chamkha · I. Pop
}

Published online: 17 April 2008

(C) Springer Science+Business Media B.V. 2008

\section{Erratum to: Transp Porous Med DOI 10.1007/s11242-005-0260-5}

1. Equations (1) and (3) should be replaced by the following equation

$$
g \beta\left(T-T_{0}\right)-\frac{1}{\rho_{0}} \frac{\partial P}{\partial X}+\frac{\mu_{\mathrm{eff}}}{\rho_{0}} \frac{\mathrm{d}^{2} U}{\mathrm{~d} Y^{2}}-\frac{v}{K} U-\frac{\rho C_{\mathrm{F}}}{\rho_{0}} U^{2}=0
$$

2. Equation (7) should be replaced by the following equation

$$
\frac{\mathrm{d}^{4} U}{\mathrm{~d} Y^{4}}=\frac{\beta g}{\alpha C_{p}}\left(\frac{\mathrm{d} U}{\mathrm{~d} Y}\right)^{2}+\frac{1}{K} \frac{\mathrm{d}^{2} U}{\mathrm{~d} Y^{2}}+\frac{\beta g}{\alpha C_{p} k} U^{2}+\frac{C_{\mathrm{F}}}{v} \frac{\mathrm{d}^{2} U^{2}}{\mathrm{~d} Y^{2}}
$$

3. Equation (10) should be replaced by the following equation

$$
R e=\frac{U_{0} D}{v} ; \quad \operatorname{Pr}=\frac{v}{\alpha} ; \quad B r=\frac{\mu U_{0}^{2}}{k \Delta T}
$$

4. The year publication to be introduced in the following two references

Srinivasan, V. and Vafai, K.: 1994, Analysis of linear encroachment in two-immiscible fluid systems, ASME J. Fluids Eng. 116, 135-139.

Vafai, K. and Kim, S.: 1989, Forced convection in a channel filled with a porous medium: an exact solution, ASME J. Heat Transfer 111, 1103-1106.

The online version of the original article can be found under doi:10.1007/s11242-005-0260-5.

J. C. Umavathi · J. P. Kumar

Department of Mathematics, Gulbarga University, Gulbarga, Karnataka 585 106, India

A. J. Chamkha

Manufacturing Engineering Department, The Public Authority for Applied Education and Training, Shuweikn 70654, Kuwait

I. Pop ( ()

Faculty of Mathematics, University of Cluj, CP 253, Cluj 3400, Romania

e-mail: popi@math.ubbcluj.ro 
5. Figures 2 to 7 should be replaced by the following graphs. Figures 8 to 10 should be removed.

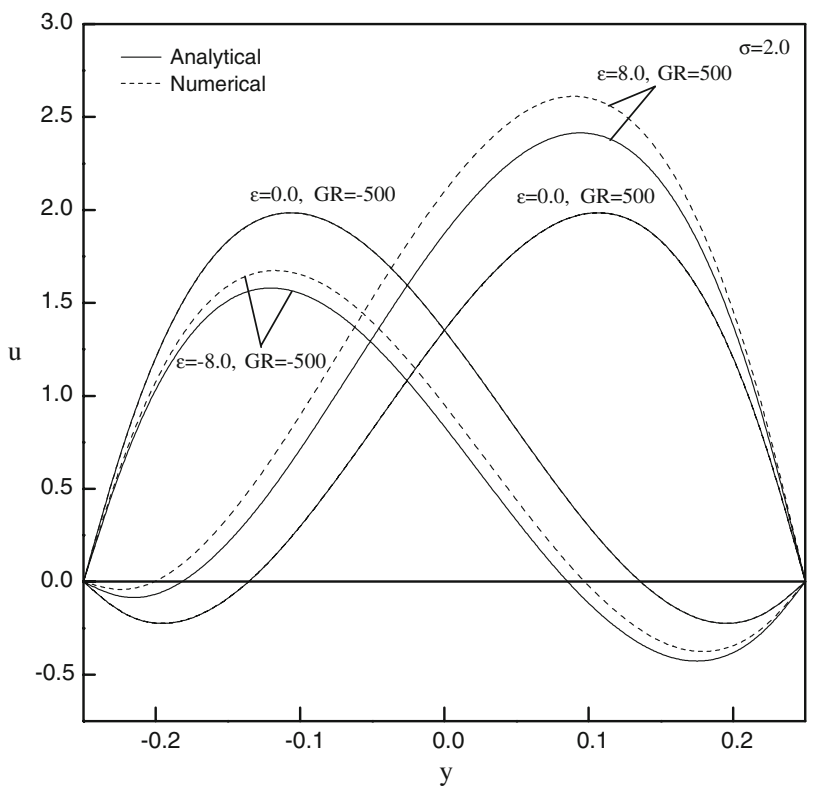

Fig. 2 Plots of $u$ versus $y$ in the case of asymmetric heating for different values of $\varepsilon$

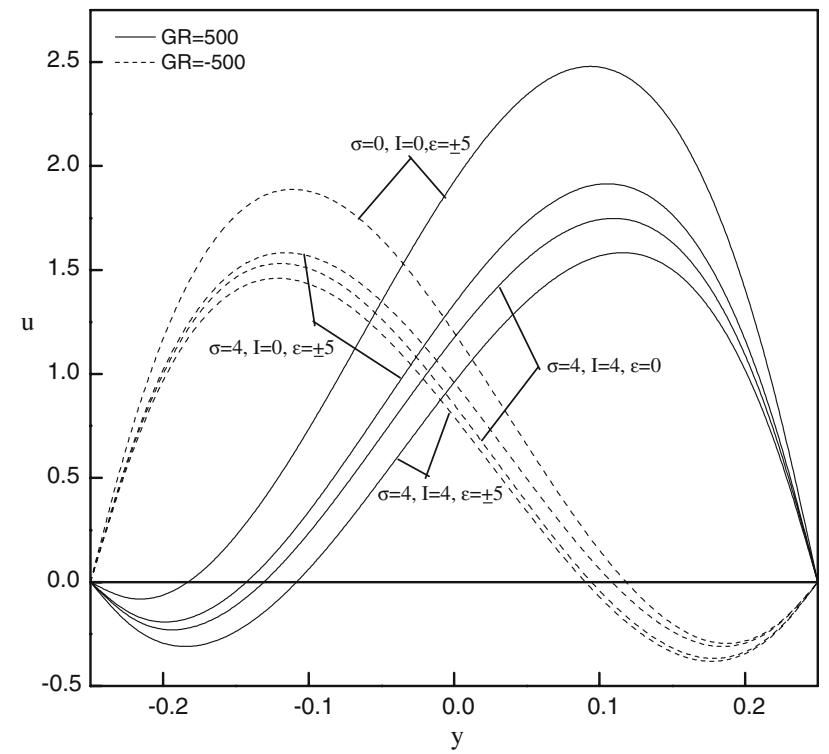

Fig. 3 Plots of velocity profiles versus y in the case of asymmetric heating for different values of $\sigma, I, \varepsilon$ and GR 


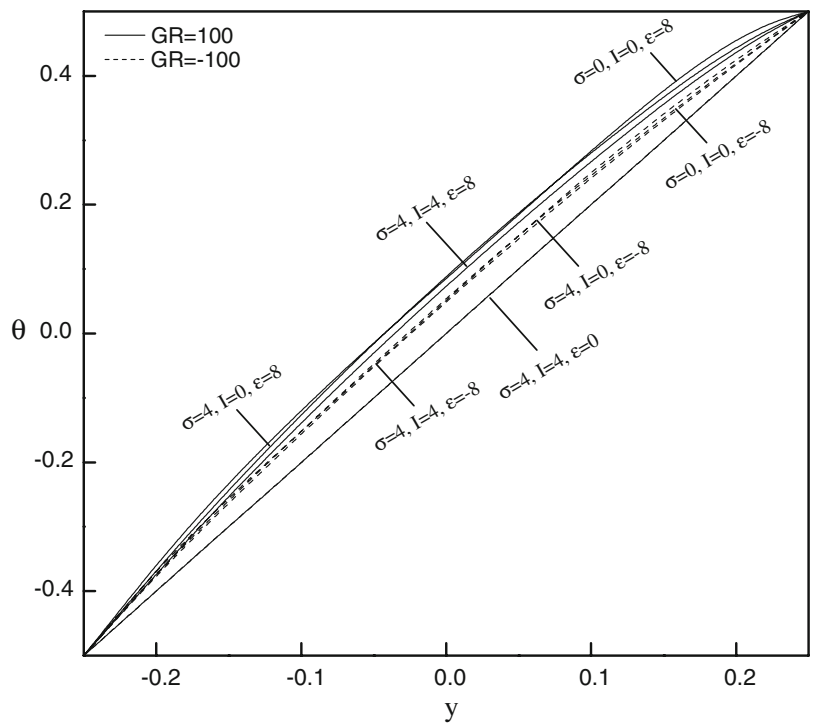

Fig. 4 Plots of temperature versus $y$ in the case of asymmetric heating for different values of $\sigma, I$, and $\varepsilon$

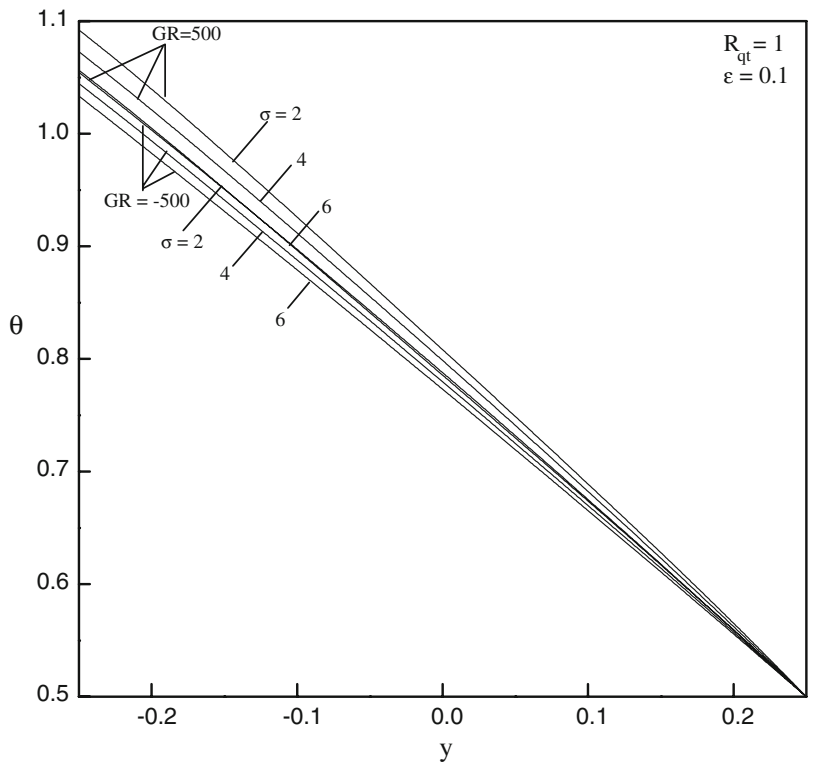

Fig. 5 Temperature profiles for different values of $\sigma$ for isoflux-isothermal case 


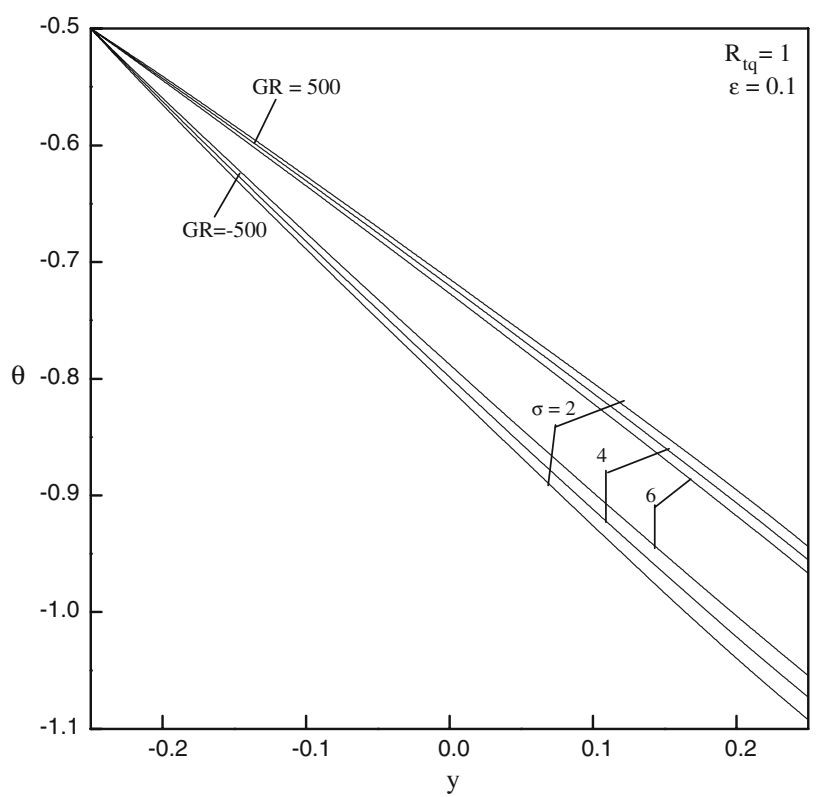

Fig. 6 Temperature profiles for different values of $\sigma$ for isothermal-isoflux case

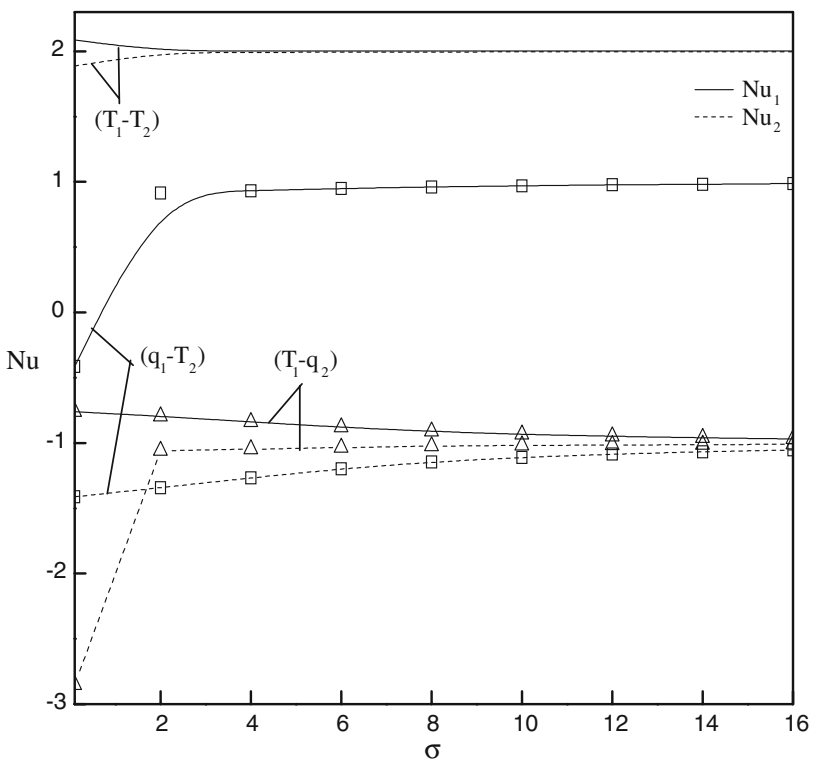

Fig. 7 Nusselt number for different values of $\sigma$ 